\title{
Association of the C47T polymorphism in SOD2 with diabetes mellitus and diabetic microvascular complications: a meta-analysis
}

\author{
C. Tian $\cdot$ S. Fang $\cdot$ X. Du $\cdot$ C. Jia
}

Received: 3 August 2010 /Accepted: 12 November 2010 / Published online: 22 December 2010

(C) Springer-Verlag 2010

\begin{abstract}
Aims/hypothesis A meta-analysis was performed to assess the association of C47T (rs4880) (also called Val16Ala) polymorphism in SOD2 gene with reduced risk of diabetes mellitus, including type 1 and type 2 diabetes, and diabetic microvascular complications (DMI) including diabetic nephropathy, diabetic retinopathy and diabetic polyneuropathy. Methods A comprehensive search was conducted to identify all case-control or cohort design studies of the abovementioned associations. The fixed or random effect pooled measure was selected on the basis of homogeneity test among studies. Heterogeneity among studies was evaluated using the $I^{2}$. Meta-regression and the 'leave one out' sensitive analysis of Patsopoulos et al. were used to explore potential sources of between-study heterogeneity. Publication bias was estimated using modified Egger's linear regression test as proposed by Harbord et al.

Results Seventeen articles were included. After excluding articles that deviated from Hardy-Weinberg equilibrium in cases and/or in controls, and were also the key contributors to between-study heterogeneity, the meta-analysis showed a significant association of the $\mathrm{C}$ allele with reduced risk of DMI in dominant (OR 0.788, 95\% CI 0.680-0.914), recessive (OR $0.808,95 \%$ CI $0.685-0.953$ ) and codominant (OR 0.828, 95\% CI 0.751-0.913) models. It also showed a significant association with reduced risk of diabetic nephropathy in the dominant model (OR $0.801,95 \%$ CI $0.664-0.967)$, and reduced risk of diabetic retinopathy in the dominant (OR $0.601,95 \%$ CI $0.423-0.855$ ), recessive
\end{abstract}

C. Tian $\cdot$ S. Fang $\cdot$ X. Du $\cdot$ C. Jia $(\triangle)$

Department of Epidemiology and Health Statistics,

Shandong University,

Jinan 250012 Shandong, People's Republic of China

e-mail: jiachongqi@sdu.edu.cn
(OR $0.548,95 \%$ CI $0.369-0.814$ ) and codominant (OR $0.651,95 \%$ CI $0.517-0.820$ ) models.

Conclusions/interpretation The meta-analysis suggested that $\mathrm{C}$ allele of C47T polymorphism in SOD2 gene has protective effects on risk of DMI, diabetic nephropathy and diabetic retinopathy. This risk needs to be confirmed by further studies.

Keywords Diabetes · Meta-analysis · Microangiopathy MnSOD · Nephropathy - Oxidative stress · Polymorphism . Polyneuropathy $\cdot$ Retinopathy $\cdot$ SOD2

$\begin{array}{ll}\text { Abbreviations } \\ \text { DMI } & \text { Diabetic microvascular complications } \\ \text { DPN } & \text { Diabetic polyneuropathy } \\ \text { FEM } & \text { Fixed effect model } \\ \text { HWE } & \text { Hardy-Weinberg equilibrium } \\ \text { MnSOD } & \text { Manganese superoxide dismutase } \\ \text { REM } & \text { Random effect model } \\ \text { SOD } & \text { Superoxide dismutase }\end{array}$

\section{Introduction}

Oxidative stress induced by superoxide anion plays a key role in the initiation as well as progression of diabetes mellitus [1] and diabetic microvascular complications (DMI) [2]. Superoxide dismutase (SOD) 2 (also known as manganese superoxide dismutase [MnSOD]) is an essential defender against mitochondrial superoxide radicals among antioxidative enzymes [3]. The $\mathrm{T}$ to $\mathrm{C}$ nucleotide polymorphism (rs4880) (previously called C47T) [4], which is located in exon 2 of SOD2 gene and results in the amino acid substitution of valine with alanine at position 16 of the protein (Val16Ala), is considered the most interesting of 
several polymorphisms in SOD2 gene, because C allele instead of $\mathrm{T}$ allele results in more efficient transport of SOD2 into the mitochondrial matrix [5], which can increase the ability to neutralise superoxide radicals. Since Chistyakov et al first showed the significant association between C47T polymorphism in SOD2 gene and risk of diabetic polyneuropathy (DPN), and no significant association with type 1 diabetes risk [6], the associations of this polymorphism with diabetes mellitus, including type 1 and type 2 diabetes, and DMI including diabetic nephropathy, diabetic retinopathy and DPN have been investigated. However, the published results are controversial. Hence we conducted a metaanalysis to: (1) assess the effect of C47T polymorphism in SOD2 gene on risk of diabetes mellitus and of DMI including diabetic nephropathy, diabetic retinopathy and DPN; (2) evaluate the potential heterogeneity among studies; and (3) explore any potential publication bias.

\section{Methods}

Search strategy A search was conducted for relevant available articles published in English or Chinese from five databases: (1) PubMed (1990-2010); (2) China National Knowledge Infrastructure (CNKI) (1990-2010); (3) Database of Chinese Scientific and Technical Periodicals (VIP) (1990-2010); (4) China Biology Medical literature database (CBM) (1990-2010); and (5) Web of Science (ISI) (19902010). The search strategy used the following keywords: 'diabetes' and 'C47T', 'Val16Ala', 'MnSOD', 'SOD2', 'manganese superoxide dismutase', 'superoxide dismutase', 'polymorphism', 'mut*' and 'varia*'. Additional studies not captured by our database searches were identified by reviewing the bibliographies of relevant articles as well as those of relevant studies.

Inclusion criteria All identified studies were carefully reviewed independently by two investigators to determine whether an individual study was eligible for inclusion in this meta-analysis. The inclusion criteria were as follows: (1) case-control or cohort study published as an original study to evaluate the association between C47T (Val16Ala) polymorphism in SOD2 gene and risk of diabetes mellitus and of DMI including diabetic nephropathy, diabetic retinopathy and DPN; (2) numbers in case and control groups or exposed and unexposed groups reported for each genotype, or data provided from which numbers could be calculated; (3) case and control groups in case-control study or exposed and unexposed groups in cohort study unrelated and drawn from the same temporally and geographically defined underlying population. If the two investigators disagreed about the eligibility of an article, it was resolved by consensus with a third reviewer.
Data extraction Data were independently extracted by two investigators who reached a consensus on all of the items. The most recent and complete article was chosen, if a study had been published more than once. Information extracted from each study was as follows: publication year, name of first author, country, ethnic origin of the studied population, number in case (exposed) and control (unexposed) groups, genotype and allele distributions, mean age, male sex percentage in case (exposed) and control (unexposed) groups.

Statistical analysis We used $\chi^{2}$ analysis with exact probability to test departure from Hardy-Weinberg equilibrium (HWE) for the C47T genotype distribution of SOD2 gene in case and control groups. Pooled measure was calculated as the inverse variance-weighted mean of the logarithm of OR with $95 \%$ CI to assess the strength of association between C47T polymorphism in SOD2 gene and risk of diabetes mellitus and of DMI including diabetic nephropathy, diabetic retinopathy and DPN for dominant $(\mathrm{CC}+\mathrm{TC}$ vs TT), recessive (CC vs $\mathrm{TC}+\mathrm{TT})$ and codominant $(\mathrm{C}$ vs $\mathrm{T})$ models, respectively. The $I^{2}$ of Higgins and Thompson was used to assess heterogeneity among studies [7]. $I^{2}$ describes the proportion of total variation attributable to between-study heterogeneity as opposed to random error or chance. In the presence of substantial heterogeneity $\left(I^{2}>50 \%\right)$ [8], the DerSimonian and Laird random effect model (REM) was adopted as the pooling method; otherwise, the fixed effect model (FEM) was used as the pooling method. Metaregression with restricted maximum likelihood estimation was performed to assess the potentially important covariates exerting substantial impact on between-study heterogeneity. The 'leave one out' sensitive analysis [9] was carried out using $I^{2}>50 \%$ as the criteria to evaluate the key studies with substantial impact on between-study heterogeneity. Publication bias was estimated using modified Egger's linear regression test, as proposed by Harbord et al [10]. An analysis of influence was conducted [11], which describes how robust the pooled estimator is to removal of individual studies. An individual study is suspected of excessive influence, if the point estimate of its omitted analysis lies outside the $95 \%$ CI of the combined analysis. All statistical analyses were performed with STATA version 9.2 (Stata Corporation, College Station, TX, USA). All reported probabilities ( $p$ values) were two-sided, with $p<0.05$ considered statistically significant.

\section{Results}

Characteristics of studies We found 17 published articles [6, 12-27] with 31 outcomes eligible for this meta-analysis on the relation of C47T polymorphism in SOD2 gene to 
diabetes mellitus and DMI including diabetic nephropathy, diabetic retinopathy and DPN. All 17 eligible studies were case-control designs. General characteristics, the C47T allele and genotype distributions in the published articles included in this meta-analysis are showed in Tables 1 and 2.

Quantitative synthesis Results of pooled analysis are summarised in detail in Table 3. This meta-analysis showed a significant protective effect of $\mathrm{C}$ allele on risk of diabetes mellitus, including type 1 and type 2 diabetes, in the recessive (REM OR $0.509,95 \%$ CI $0.300-0.861$ ) and codominant (REM OR $0.672,95 \%$ CI $0.459-0.984$ ) models, but this was only marginally significant in the dominant model (REM OR 0.626, 95\% CI 0.391-1.002). However, no significant association was found between $\mathrm{C}$ allele and reduced risk of diabetes mellitus after excluding articles that deviated from HWE in cases and/or in controls for dominant (REM OR 0.594, 95\% CI 0.325-1.086), recessive (REM OR $0.430,95 \%$ CI $0.162-1.141$ ) and codominant (REM OR $0.637,95 \%$ CI $0.371-1.093$ ) models.

The $\mathrm{C}$ allele was found to be significantly associated with a reduced risk of diabetic nephropathy in the dominant (REM OR 0.734, 95\% CI 0.559-0.964) model, with marginal significance in codominant (REM OR 0.820, 95\% CI 0.670-1.003) models, and no significant association in the recessive model (REM OR 0.948, 95\% CI 0.725-1.239). After exclusion of articles deviating from HWE in cases and/or in controls, the associations in the above-mentioned inherited models were not significant.

With regard to diabetic retinopathy, no significant association with the $\mathrm{C}$ allele was found in dominant (REM OR 0.685, 95\% CI 0.444-1.057), recessive (REM OR $0.773,95 \%$ CI $0.408-1.463$ ) and codominant (REM OR $0.759,95 \%$ CI $0.503-1.147$ ) models. After exclusion of articles deviating from HWE in cases and/or in controls, the associations in the above-mentioned inherited models remained non-significant.

The meta-analysis also showed a significant association between the $\mathrm{C}$ allele and reduced risk of DPN in dominant (FEM OR 0.197, 95\% CI 0.101-0.383), recessive (FEM OR $0.367,95 \%$ CI $0.246-0.549$ ) and codominant (FEM OR $0.506,95 \%$ CI $0.397-0.644)$ models. However, all relevant articles regarding the association between $\mathrm{C} 47 \mathrm{~T}$ polymorphism and risk of DPN deviated from HWE in cases and/or in controls.

In addition, when we combined diabetic nephropathy, diabetic retinopathy and DPN into overall DMI, we found a significant effect of $\mathrm{C}$ allele on reduced risk of DMI in dominant (REM OR 0.649 , 95\% CI $0.520-0.809$ ), recessive (REM OR $0.733,95 \%$ CI $0.573-0.940$ ) and codominant (REM OR 0.739, 95\% CI 0.631-0.866) models. However, no significant association was found between $\mathrm{C}$ allele and reduced risk of DMI after excluding articles that deviated from HWE in cases and/or in controls with regard to the above-mentioned inherited models.

Sources of heterogeneity and sensitive analysis As seen in Table 3, before the 'leave one out' sensitive analysis [9], strong evidence of heterogeneity among studies was demonstrated in the above-mentioned inherited models for risk of diabetes mellitus, DMI, diabetic nephropathy and diabetic retinopathy, with the exception in the recessive model for diabetic nephropathy risk. No significant heterogeneity among studies was found in the above-mentioned inherited models for DPN risk.

After excluding the articles deviating from HWE in cases and/or in controls, univariate meta-regression analysis, with the covariates publication year, ethnicity (categorised as Europeans, East Asians, South Asians and North Africans), sex (ratio of males in per cent in case group to that in control group), age (ratio of mean age in case group to that in control group) and sample size, showed that no covariates had a significant impact on between-study heterogeneity.

The key contributors of the articles to between-study heterogeneity assessed by the 'leave one out' sensitive analysis [9] are presented as 'articles excluded' in Table 3.

After exclusion of articles that deviated from HWE in cases and/or in controls, and were the key contributors to between-study heterogeneity, the meta-analysis showed a significant association of the $\mathrm{C}$ allele with reduced risk of DMI in dominant (OR 0.788, 95\% CI 0.680-0.914) (Fig. 1), recessive (OR $0.808,95 \%$ CI $0.685-0.953$ ) and codominant (OR $0.828,95 \%$ CI $0.751-0.913$ ) models. It also showed a significant association of the $\mathrm{C}$ allele with reduced risk of diabetic nephropathy in the dominant model (OR 0.801, 95\% CI 0.664-0.967) (Fig. 2), as well as with reduced risk of diabetic retinopathy in dominant (OR $0.601,95 \% \mathrm{CI}$ $0.423-0.855$ ), recessive (OR $0.548,95 \%$ CI $0.369-0.814$ ) and codominant (OR $0.651,95 \%$ CI $0.517-0.820)$ models.

Influence analysis After excluding articles that deviated from HWE in cases and/or in controls, and were the key contributors to between-study heterogeneity, no individual study was found to have excessive influence on the pooled effect in the above-mentioned inherited models with regard to diabetes mellitus, DMI, diabetic nephropathy and diabetic retinopathy, with the exception of one study [19] on reduced risk of diabetes mellitus in the recessive model (OR of this study was 0.701 , with $95 \%$ CI for pooled effect of $0.757-1.452$ ).

Publication bias evaluation After excluding articles that deviated from HWE in cases and/or in controls, and were the key contributors to between-study heterogeneity, no significant publication bias was detected in the abovementioned inherited models for diabetes mellitus, DMI, 


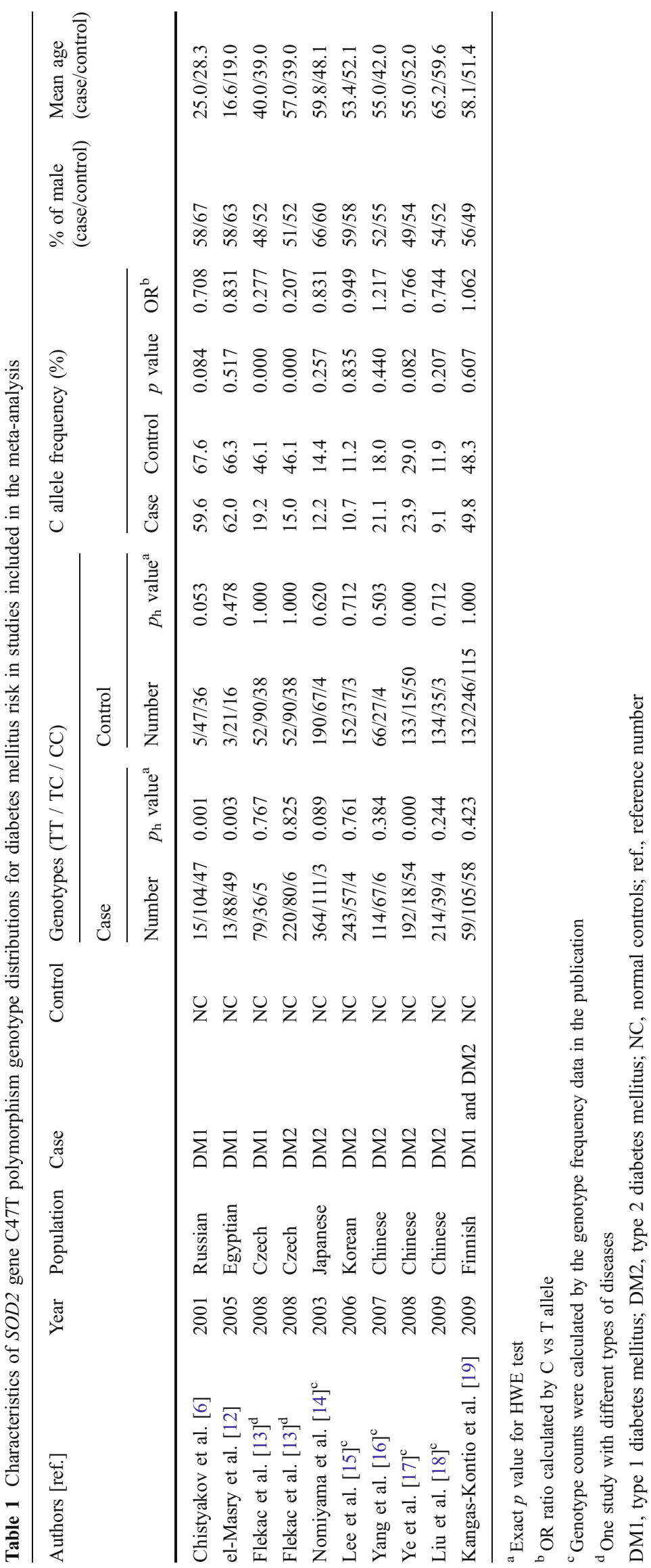




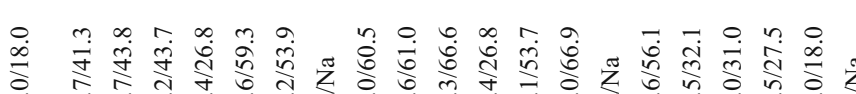

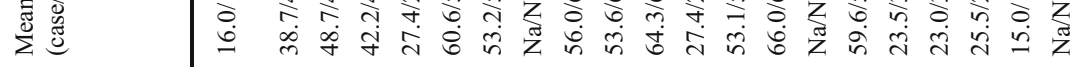

竞

范总

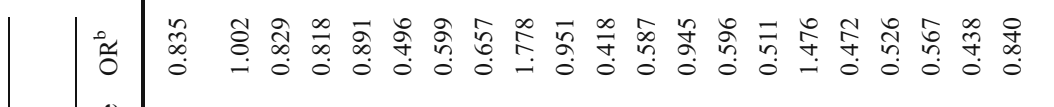

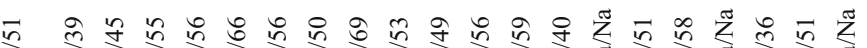

तु के 穴 तु

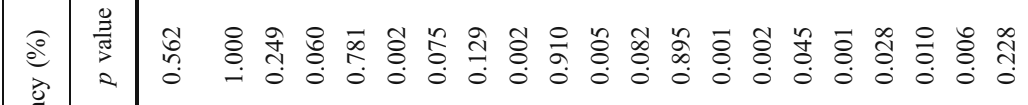

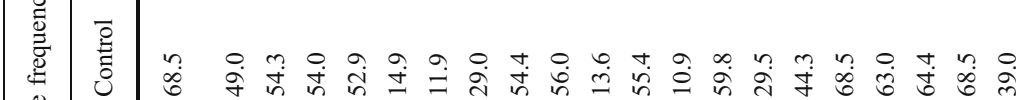

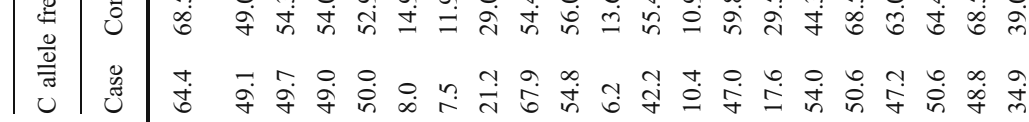

-

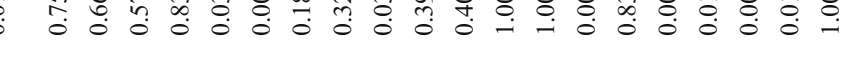

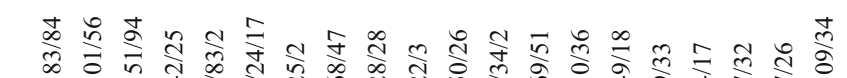

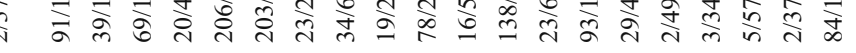

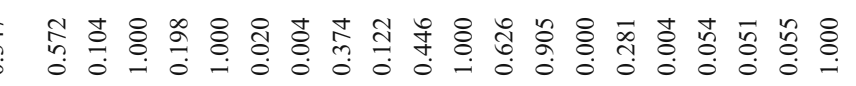

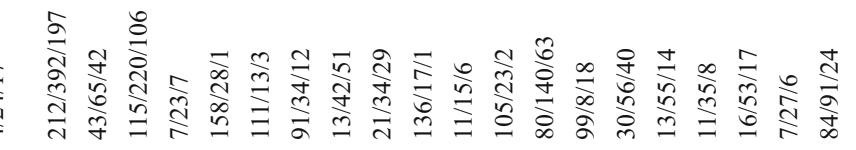

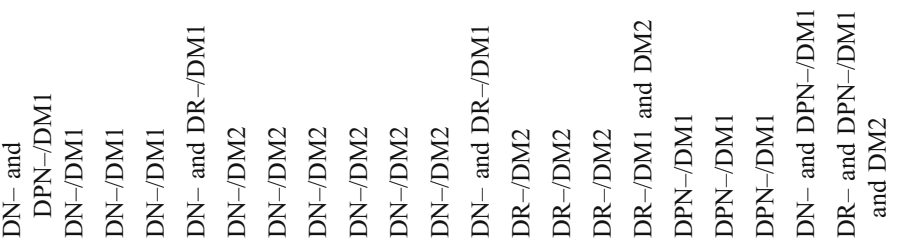

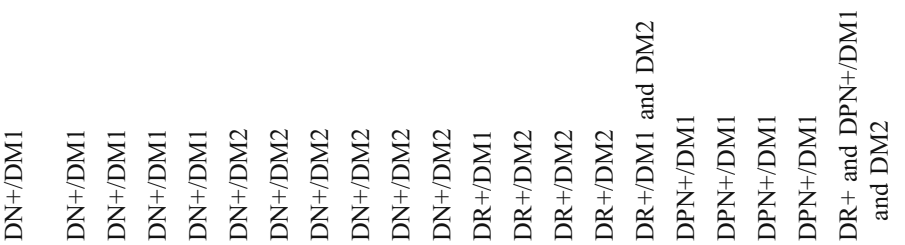

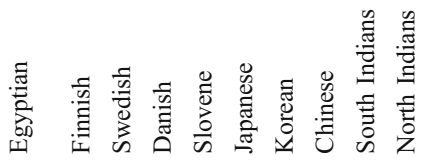

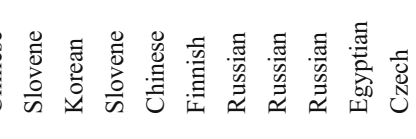

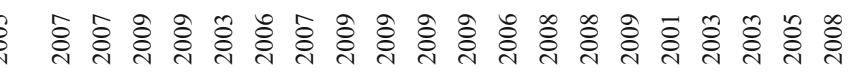

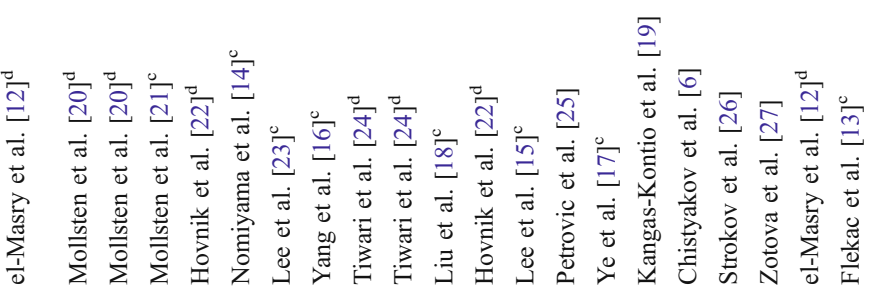

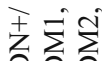

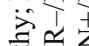

要藏

突艺

空声言

을

证.

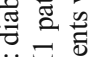

芸方䒿

窟主

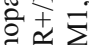

定

总公

ब핳

定

苍突

응 त्ञ

츤 㻤

ํํㄹ

哥主

它合

究台

毒泀

过

氜

뉼.

(c)

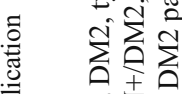

空方方

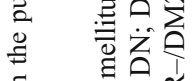

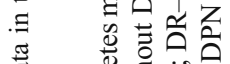

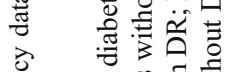

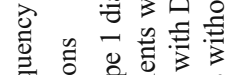

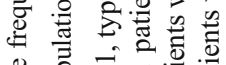

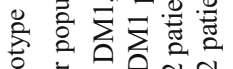

总

๘

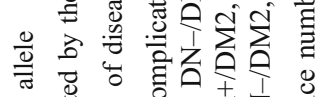

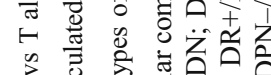

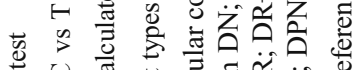

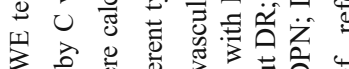

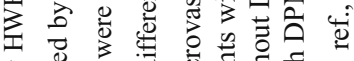

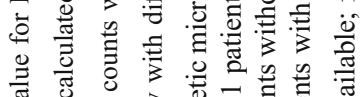

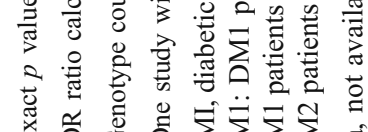

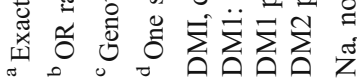




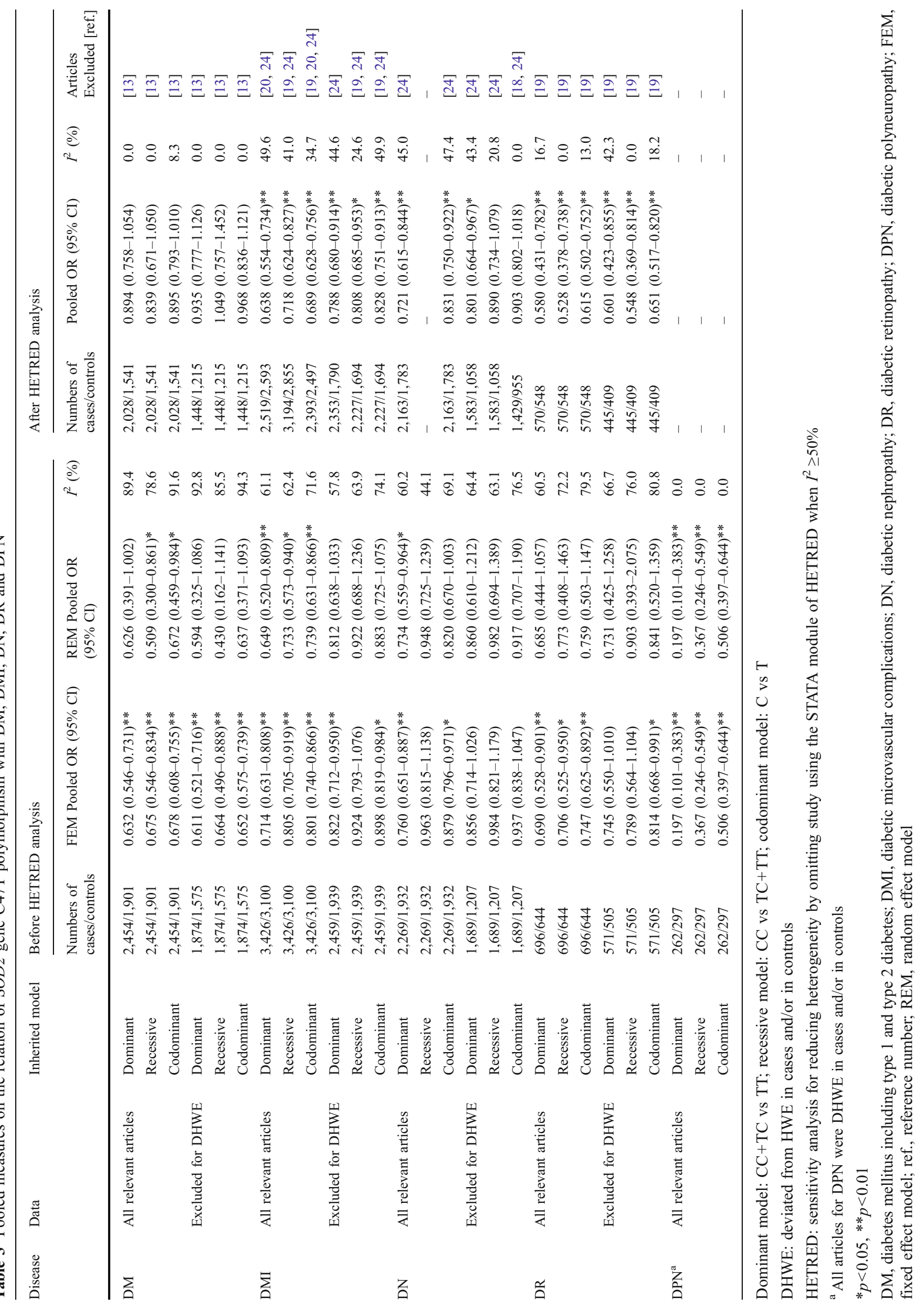


Fig. 1 Forest plot of ORs for $\mathrm{DMI}$ in dominant model (CC+ TC vs TT) of SOD2 gene C47T polymorphism. White diamond denotes the pooled OR. Black squares indicate the OR in each study, with square sizes inversely proportional to the standard error of the OR. Horizontal lines represent $95 \%$ CI. ${ }^{a}$ One study with different types of diseases or populations. Ref., reference number

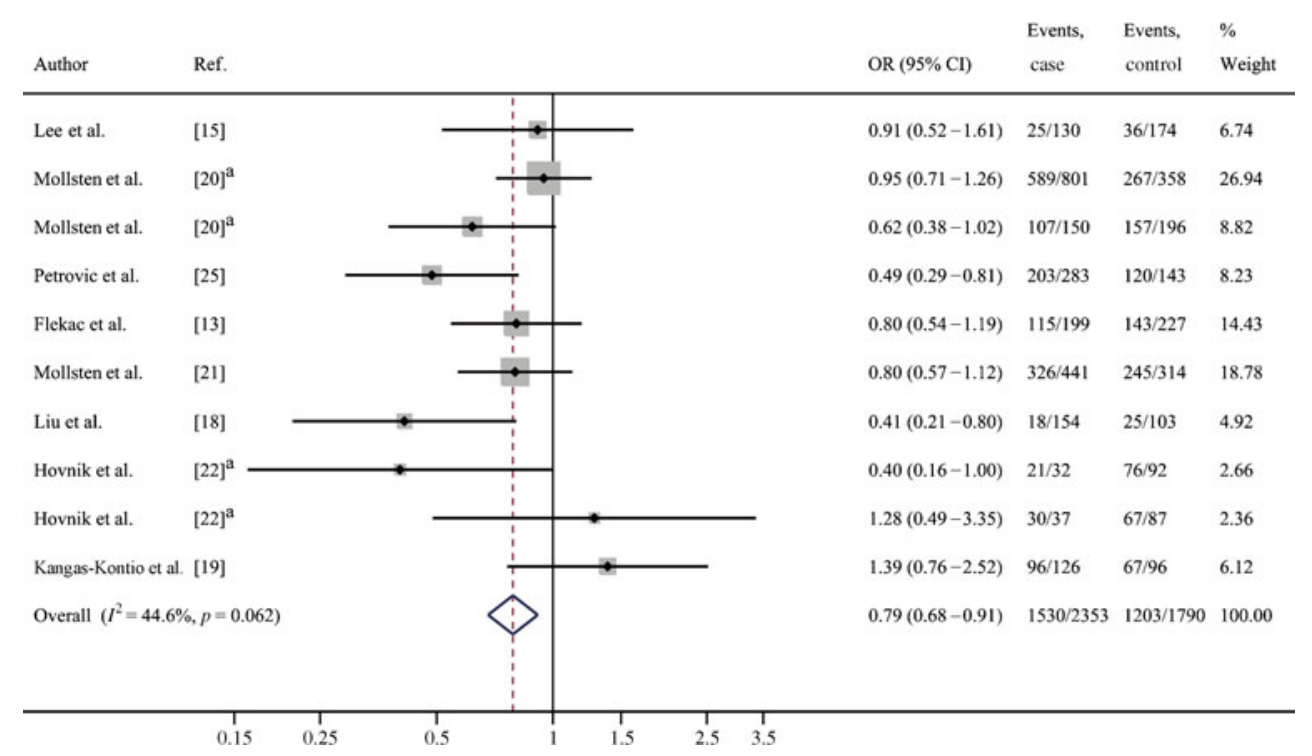

diabetic nephropathy and diabetic retinopathy (data not shown).

\section{Discussion}

Clinical and experimental studies indicate that oxidative stress is associated with diabetes mellitus [1], diabetic nephropathy [28], diabetic retinopathy [29] and DPN [30]. The SOD2 encoded by the $\mathrm{T}$ allele, which disrupts the alpha-helix structure of the targeting sequence, is retained in the mitochondrial inner membrane and induces 30 to $40 \%$ lower activity and oxidative stress level. Chistyakov et al [6] first showed the significant protective effect of C47T polymorphism in SOD2 gene on DPN risk in 82 DPN cases and 84 non-DPN controls in type 1 diabetes mellitus patients, but no association with risk of type 1 diabetes mellitus was found in 166 type 1 diabetes mellitus cases and 88 normal controls in a Russian population. Nevertheless, results of subsequent studies about the effect of this polymorphism on risk of diabetes mellitus, including type 1 and type 2 diabetes, and of DMI including diabetic nephropathy, diabetic retinopathy and DPN were conflicting.

Because of the above-mentioned inconsistent results from relatively small studies underpowered to detect the effect, a meta-analysis is the appropriate approach to obtain a more definitive conclusion regarding the role of SOD2 gene C47T polymorphism on risk of diabetes mellitus and DMI including diabetic nephropathy, diabetic retinopathy and DPN. Our meta-analysis, of 17 published articles [6, 12-27] with 31 outcomes from different ethnic origins, allowed a much greater possibility of reaching reasonably strong conclusions.

As mentioned in one paper [31], between-study heterogeneity is common in meta-analysis for genetic association studies. Our meta-analysis also showed significant between-study heterogeneity in most of the inherited models regarding diabetes mellitus, DMI, diabetic nephrop-
Fig. 2 Forest plot of ORs for diabetic nephropathy in dominant model (CC+TC vs TT) of SOD2 gene C47T polymorphism. White diamond denotes the pooled OR. Black squares indicate the OR in each study, with square sizes inversely proportional to the standard error of the OR. Horizontal lines represent $95 \%$ CI. ${ }^{a}$ One study with different types of populations. Ref., reference number

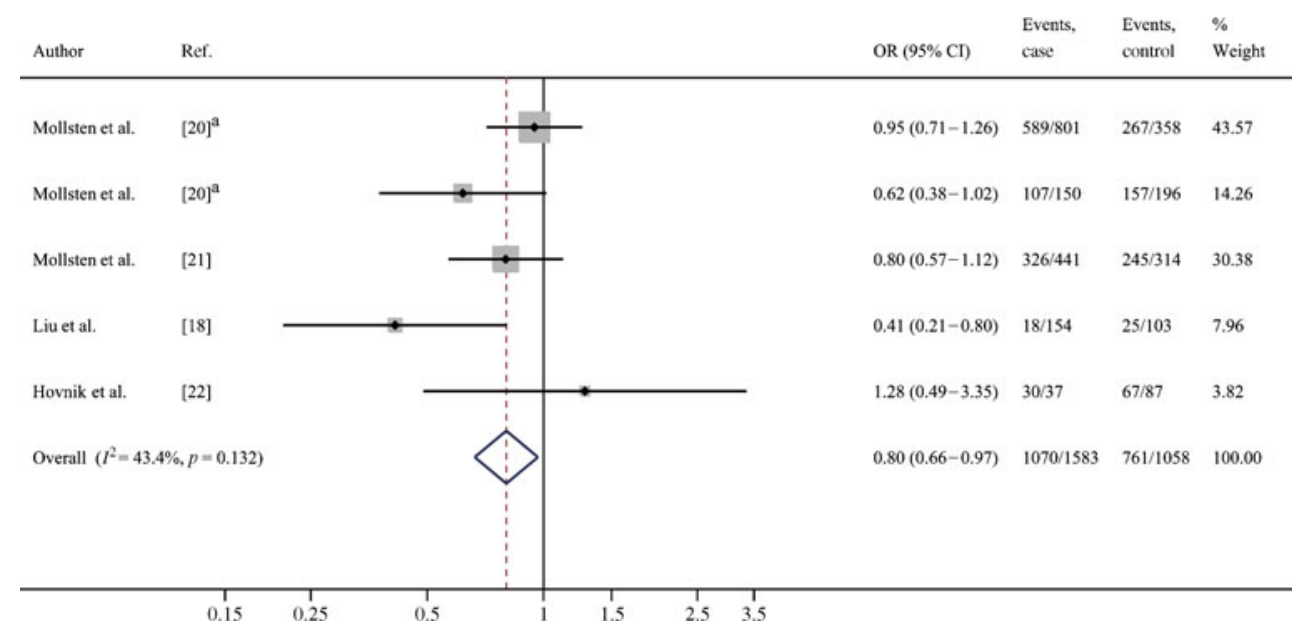


athy and diabetic retinopathy. An indeterminate number of characteristics that vary among studies could be the cause of between-study heterogeneity, e.g. design quality, population stratification, characteristics of the sample, noncomparable measure of genotyping, variation of the covariate, deviation from HWE in some studies, etc. Thus we used meta-regression and 'leave one out' sensitive analysis [9], which aims to reduce between-study heterogeneity and explore the potential important causes of between-study heterogeneity for both covariates and studies. Our meta-analysis of the studies that were in HWE in cases and controls did not identify any of the aforementioned covariates as being an important contributor to between-study heterogeneity. When, after the 'leave one out' sensitive analysis using $I^{2}>50 \%$ as the criteria, we performed data analysis on the studies that were in HWE in cases and controls, our results showed that the $\mathrm{C}$ allele of C47T polymorphism in SOD2 gene had a significant effect on reduced risk of DMI, diabetic nephropathy and diabetic retinopathy, but not of diabetes mellitus.

According to another article [32] and the SNP database at the NIH (www.ncbi.nlm.nih.gov/projects/SNP/snp_ref. cgi?rs=4880, accessed 24 September 2010), the $C$ allele frequency in the Asian population is significantly lower than in most European populations. Such heterogeneous genetic backgrounds might be, in part, responsible for the heterogeneity of effect on the disease across the ethnicity, even though we could not detect this in our meta-analysis. Diabetes mellitus and DMI including diabetic nephropathy, diabetic retinopathy and DPN, have a complex aetiology and pathophysiology generated by the combined effects of genes and environment factors. Although ethnicity, sex and age were not found to be important sources of disease-effect heterogeneity across the studies in this meta-analysis, other genetic and environment variables, as well as their possible interaction, may well be potential contributors to this disease-effect unconformity. In this respect, the lack of relevant study-level covariates in the reported articles precluded a more robust assessment of sources of this heterogeneity. Other possibilities related to disease-effect diversity, such as variations in design quality, variations in genotyping, etc. could not be ruled out.

In this meta-analysis, we could not find any significant publication bias in the above-mentioned inherited models for diabetes mellitus, including type 1 and type 2 diabetes, DMI, diabetic nephropathy and diabetic retinopathy, this may be due to the small number of studies in those metaanalyses, especially for diabetic retinopathy risk evaluation.

In conclusion, this meta-analysis suggested that the $\mathrm{C}$ allele of C47T polymorphism in SOD2 gene had significant protective effects on risk of DMI, diabetic nephropathy and diabetic retinopathy. Since potential biases and confounders could not be ruled out completely in this meta-analysis, further studies are needed to confirm these results.
Acknowledgements This study was sponsored by a grant from Shandong Provincial Natural Science Foundation, China (Y2007C005).

Duality of interest The authors declare that there is no duality of interest associated with this manuscript.

\section{References}

1. Valko M, Leibfritz D, Moncol J, Cronin MT, Mazur M, Telser J (2007) Free radicals and antioxidants in normal physiological functions and human disease. Int J Biochem Cell Biol 39:44-84

2. Kiritoshi S, Nishikawa T, Sonoda K et al (2003) Reactive oxygen species from mitochondria induce cyclooxygenase- 2 gene expression in human mesangial cells: potential role in diabetic nephropathy. Diabetes 52:2570-2577

3. McIntyre M, Bohr DF, Dominiczak AF (1999) Endothelial function in hypertension: the role of superoxide anion. Hypertension 34:539-545

4. Rosenblum JS, Gilula NB, Lerner RA (1996) On signal sequence polymorphisms and diseases of distribution. Proc Natl Acad Sci U S A 93:4471-4473

5. Sutton A, Khoury H, Prip-Buus C, Cepanec C, Pessayre D, Degoul F (2003) The Ala16Val genetic dimorphism modulates the import of human manganese superoxide dismutase into rat liver mitochondria. Pharmacogenetics 13:145-157

6. Chistyakov DA, Savost'anov KV, Zotova EV, Nosikov VV (2001) Polymorphisms in the Mn-SOD and EC-SOD genes and their relationship to diabetic neuropathy in type 1 diabetes mellitus. BMC Med Genet 2:4

7. Higgins JP, Thompson SG (2002) Quantifying heterogeneity in a meta-analysis. Stat Med 21:1539-1558

8. Higgins JP, Thompson SG, Deeks JJ, Altman DG (2003) Measuring inconsistency in meta-analyses. BMJ 327:557-560

9. Patsopoulos NA, Evangelou E, Ioannidis JP (2008) Sensitivity of between-study heterogeneity in meta-analysis: proposed metrics and empirical evaluation. Int J Epidemiol 37:1148-1157

10. Harbord RM, Egger M, Sterne JA (2006) A modified test for small-study effects in meta-analyses of controlled trials with binary endpoints. Stat Med 25:3443-3457

11. Tobias A (1999) Assessing the influence of a single study in the meta-analysis estimate. Stata Tech Bull 47:15-17

12. el-Masry TM, Zahra MA, el-Tawil MM, Khalifa RA (2005) Manganese superoxide dismutase alanine to valine polymorphism and risk of neuropathy and nephropathy in Egyptian type 1 diabetic patients. Rev Diabet Stud 2:70-74

13. Flekac M, Skrha J, Hilgertova J, Lacinova Z, Jarolimkova M (2008) Gene polymorphisms of superoxide dismutases and catalase in diabetes mellitus. BMC Med Genet 9:30

14. Nomiyama T, Tanaka Y, Piao L et al (2003) The polymorphism of manganese superoxide dismutase is associated with diabetic nephropathy in Japanese type 2 diabetic patients. J Hum Genet 48:138-141

15. Lee SJ, Choi MG (2006) Association of manganese superoxide dismutase gene polymorphism (V16A) with diabetic macular edema in Korean type 2 diabetic patients. Metabolism 55:16811688

16. Yang Y, Song DP, Wang YM (2007) Association between manganese superoxide dismutase gene V16A polymorphism and type 2 diabetic nephropathy. Chin J Diabetes 15:18-20

17. Ye LX, Yang MP, Qiu H, Guo KQ, Yan JS (2008) Association of the polymorphism in manganese superoxide dismutase gene with diabetic retinopathy in Chinese type 2 diabetic patients. Zhonghua Yi Xue Yi Chuan Xue Za Zhi 25:452-454 
18. Liu L, Zheng T, Wang N et al (2009) The manganese superoxide dismutase Val16Ala polymorphism is associated with decreased risk of diabetic nephropathy in Chinese patients with type 2 diabetes. Mol Cell Biochem 322:87-91

19. Kangas-Kontio T, Vavuli S, Kakko SJ et al (2009) Polymorphism of the manganese superoxide dismutase gene but not of vascular endothelial growth factor gene is a risk factor for diabetic retinopathy. Br J Ophthalmol 93:1401-1406

20. Mollsten A, Marklund SL, Wessman M et al (2007) A functional polymorphism in the manganese superoxide dismutase gene and diabetic nephropathy. Diabetes 56:265-269

21. Mollsten A, Jorsal A, Lajer M, Vionnet N, Tarnow L (2009) The V16A polymorphism in SOD2 is associated with increased risk of diabetic nephropathy and cardiovascular disease in type 1 diabetes. Diabetologia 52:2590-2593

22. Hovnik T, Dolzan V, Bratina NU, Podkrajsek KT, Battelino T (2009) Genetic polymorphisms in genes encoding antioxidant enzymes are associated with diabetic retinopathy in type 1 diabetes. Diabetes Care 32:2258-2262

23. Lee SJ, Choi MG, Kim DS, Kim TW (2006) Manganese superoxide dismutase gene polymorphism (V16A) is associated with stages of albuminuria in Korean type 2 diabetic patients. Metabolism 55:1-7

24. Tiwari AK, Prasad P, Thelma BK et al (2009) Oxidative stress pathway genes and chronic renal insufficiency in Asian Indians with type 2 diabetes. J Diabetes Complications 23:102-111

25. Petrovic MG, Cilensek I, Petrovic D (2008) Manganese superoxide dismutase gene polymorphism (V16A) is associated with diabetic retinopathy in Slovene (Caucasians) type 2 diabetes patients. Dis Markers 24:59-64

26. Strokov IA, Bursa TR, Drepa OI, Zotova EV, Nosikov VV, Ametov AS (2003) Predisposing genetic factors for diabetic polyneuropathy in patients with type 1 diabetes: a populationbased case-control study. Acta Diabetol 40(Suppl 2):S375S379

27. Zotova EV, Chistiakov DA, Savost'ianov KV et al (2003) Association of the SOD2 Ala(-9)Val and SOD3 Arg213Gly polymorphisms with diabetic polyneuropathy in patients with diabetes mellitus type 1. Mol Biol (Mosk) 37:404-408

28. Niedowicz DM, Daleke DL (2005) The role of oxidative stress in diabetic complications. Cell Biochem Biophys 43:289-330

29. Camera A, Hopps E, Caimi G (2007) Diabetic microangiopathy: physiopathological, clinical and therapeutic aspects. Minerva Endocrinol 32:209-229

30. Rosen P, Nawroth PP, King G, Moller W, Tritschler HJ, Packer L (2001) The role of oxidative stress in the onset and progression of diabetes and its complications: a summary of a Congress Series sponsored by UNESCO-MCBN, the American Diabetes Association and the German Diabetes Society. Diabetes Metab Res Rev 17:189-212

31. Munafo MR, Flint J (2004) Meta-analysis of genetic association studies. Trends Genet 20:439-444

32. Van Landeghem GF, Tabatabaie P, Kucinskas V, Saha N, Beckman G (1999) Ethnic variation in the mitochondrial targeting sequence polymorphism of MnSOD. Hum Hered 49:190-193 